\title{
Nanostructured Natural-Based Polyelectrolyte Multilayers to Agglomerate Chitosan Particles into Scaffolds for Tissue Engineering
}

\author{
Emanuel Sá Miranda, M.Sc., ${ }^{1,2}$ Tiago H. Silva, Ph.D.,, ${ }^{1,2}$ Rui L. Reis, Ph.D., D.Sc., ${ }^{1,2}$ and João F. Mano, Ph.D. ${ }^{1,2}$
}

The layer-by-layer $(\mathrm{LbL})$ deposition technique is a self-assembly process that allows the coating of material's surface with nanostructured layers of polyelectrolytes, allowing to control several surface properties. This technique presents some advantages when compared with other thin film assembly techniques, like having the possibility to coat surfaces with complex geometries in mild conditions or to incorporate active compounds. Tissue engineering (TE) involves typically the use of porous biodegradable scaffolds for the temporary support of cells. Such structures can be produced by agglomeration of microspheres that needs to be fixed into a three-dimensional (3D) structure. In this work we suggest the use of LbL to promote such mechanical fixation in free-formed microspheres assemblies and simultaneously to control the properties of its surface. For the proof of concept the biological performance of chitosan/alginate multilayers is first investigated in two-dimensional (2D) models in which the attachment and proliferation of L929 and ATDC5 cells are studied in function of the number of layers and the nature of the final layer. Scaffolds prepared by agglomeration of chitosan particles using the same multilayered system were processed and characterized; it was found that they could support the attachment and proliferation of ATDC 5 cells. This study suggests that LbL can be used as a versatile methodology to prepare scaffolds by particle agglomeration that could be suitable for TE applications.

\section{Introduction}

O RGAN AND TISSUE LOSS or failure from an injury, disease, or other type of damage is a major human health problem. Organ or tissue transplantation or substitution by prostheses and other medical devices are among the present proposed strategies to solve that problem, but without total success due to donor shortage or to the inability to replace all the functions of the damaged tissue or organ. Tissue engineering (TE) has thus emerged as an alternative approach to treat the loss or malfunction of a tissue without the limitations of current therapies since it aims the total regeneration of the damaged tissue or organ. ${ }^{1-3}$

Scaffolds are considered an essential element in many TE strategies. Several methods have been used to prepare such interconnected porous structures, ${ }^{4,5}$ as for instance foaming, ${ }^{6,7}$ fiber extrusion and bonding, ${ }^{8,9}$ three-dimensional (3D) printing, ${ }^{10,11}$ phase separation, ${ }^{12,13}$ emulsion freeze-drying, ${ }^{14}$ porogen leaching, ${ }^{15-17}$ in situ pore forming, ${ }^{18}$ particle aggregation, ${ }^{19,20}$ electrospinning, ${ }^{21}$ supercritical fluids technology, ${ }^{22,23}$ and combinations of particles and cells. ${ }^{24}$
Particles agglomeration has been proposed to develop scaffolds, based on the physical or chemical aggregation of particles. ${ }^{19,20,25,26}$ The pore size of this type of scaffold is controlled by the particle diameter, due to the interstices formed when the particles are aggregated. One drawback is that when using microparticles, pore size may be very low. However, this type of scaffolds can exhibit very promising mechanical properties, depending on the materials used and the processing conditions and the interconnectivity of the pores is assured. Further, mechanical properties can be improved with the incorporation of a reinforcement phase (namely, a ceramic). The greatest advantage of this type of scaffolds is probably the possibility to incorporate high quantities of molecules inside the particles, with the particles being used not only to provide a support for cell adhesion but also as carriers for the controlled delivery of bioactive agents, such as growth or differentiation factors, to manipulate cellular processes within the scaffold and in implantation place. ${ }^{27}$

In the general TE strategy, the materials used on the development of scaffolds should be nontoxic and biocompatible

${ }^{1} 3 \mathrm{~B}$ 's Research Group-Biomaterials, Biodegradables and Biomimetics, University of Minho, Headquarters of the European Institute of Excellence on Tissue Engineering and Regenerative Medicine, Taipas, Guimarães, Portugal.

${ }^{2}$ ICVS/3B's, PT Government Associate Laboratory, Braga/Guimarães, Portugal. 
and promote favorable cellular interactions and tissue development, but also biodegradable and bioresorbable, to support the reconstruction of a new tissue without inflammation. Among the different materials that have been proposed, natural-based polymers are one of the most attractive options, mainly due to their similarities to biological macromolecules, which the biological environment is prepared to recognize and deal with metabolically. ${ }^{28}$ Being similar to the extracellular matrix, they may also avoid toxicity, inflammation, or immunological reactions, often observed with synthetic polymers. Particles agglomerated scaffolds have been prepared using cross-linked chitosan particles by pressing them into molds and heating the resulting constructs at $50^{\circ} \mathrm{C}$ during a few days. ${ }^{20}$ It was also shown that chitosan particles could agglomerate when combined with cells, as cells could be linked to more than one particle. ${ }^{24}$

In this work, we propose a completely new way to join free-formed assembly of particles into 3D scaffolds that could take place in mild conditions, using layer-by-layer (LbL) technology.

LbL technology has been proposed by Decher and coworkers ${ }^{29,30}$ and is based on the deposition of polyelectrolytes of opposite charge in an alternate fashion that self-assemble and self-organize on the material's surface, rendering polyelectrolyte multilayered films. This versatile technique has attracted an increasing number of researchers in recent years, due to its wide range of advantages for biomedical applications, such as the ease of preparation under physiological compatible conditions, possibility to incorporate biopolymers, bioactive molecules, and extracellular matrix components in the films, tunable mechanical properties, and spatial-temporal control over film organization. ${ }^{31-33}$

Most of the works that have been performed using LbL technology are devoted to the build-up of these multilayered films onto flat substrates. In this work, we intend to transpose this methodology toward the 3D level, by using LbL deposition technique to join particles together. The concept is based on the formation of thin nanostructured films onto initially packed particles in any given geometry, to obtain an interconnected porous structure in which the particles are physically held together by the multilayered coatings. This could allow in a single method to agglomerate particles and modify the surface of the scaffold through the selection of polyelectrolytes with the desired chemical motifs.

For the proof of concept, particles of chitosan cross-linked with genipin are joined together with alginate/chitosan multilayers. The buildup of such multilayered structure film onto flat surfaces was previously characterized by quartz crystal microbalance and Fourier transform infrared spectroscopy. ${ }^{34}$ In this work, a preliminary evaluation of biological performance of developed scaffolds was assessed by culturing ATDC5 cells in particles agglomerated by 10 and 20 bilayers of alginate and chitosan.

\section{Materials and Methods}

\section{Materials}

Chitosan (medium molecular weight, 87\% deacetylation) was purchased from Sigma-Aldrich and submitted to a purification process by repricipitation. Alginic acid and sodium salt from brown algae was purchased from Sigma-Aldrich and used as received.

\section{LbL assembly in two-dimensional surfaces}

The LbL deposition onto flat surfaces was performed in 13-mm-diameter glass coverslips (Agar Scientific). The coverslips were placed in a sonicating solution for $15 \mathrm{~min}$ after which they were rinsed with purified water. This procedure was performed twice for the same samples that were then rinsed with ethanol and finally dried by using nitrogen. For the LbL coating, chitosan was dissolved in a $1 \%$ acetic acid $0.15 \mathrm{M} \mathrm{NaCl}$ solution and left overnight at room temperature. Alginate was dissolved in a $0.15 \mathrm{M} \mathrm{NaCl}$ solution and left overnight at room temperature. Both solutions had a polyelectrolyte concentration of $0.1 \% \mathrm{w} / \mathrm{v}$. The glass coverslips have a negative surface charge and so, for the LbL assembly, the chitosan solution was the first to be used in the dipping process. Coverslips were dipped in the chitosan solution for $15 \mathrm{~min}$, followed by $5 \mathrm{~min}$ in purified water. Then, the coverslips were dipped in the alginate solution for $15 \mathrm{~min}$, followed by $5 \mathrm{~min}$ in purified water. After these four steps one double layer (DL) was assembled. The process was repeated until $10 \mathrm{DL}$ (alginate in the terminal layer), $101 / 2 \mathrm{DL}$ (chitosan in the terminal layer), $20 \mathrm{DL}$, and 201/2 DL were assembled. After the LbL deposition, the coverslips were dried using nitrogen.

\section{Chitosan particles production}

Chitosan was dissolved overnight in an acetic acid solution $(1 \% \mathrm{v} / \mathrm{v})$ to obtain a $2 \% \mathrm{w} / \mathrm{v}$ chitosan solution. After complete dissolution, the solution was extruded using a syringe, at a constant rate of $3 \mu \mathrm{L} / \mathrm{s}$, to form chitosan droplets into a previously prepared $1 \mathrm{M} \mathrm{NaOH}$ precipitation bath, under stirring, where $1 \mathrm{~mm}$ particles were formed. Chitosan particles were left in the precipitation bath overnight to become more stable. Particles were then repeatedly washed with distilled water and placed in a $0.3 \% \mathrm{w} / \mathrm{v}$ genipin (Wako) cross-linking bath under stirring for about $2 \mathrm{~h}$, after which they were repeatedly washed with distilled water. ${ }^{35}$ The particles were then removed, placed into Petri dishes, and left to dry overnight in a vacuum oven, at room temperature. After drying, particle diameter was significantly reduced from $1 \mathrm{~mm}$ to $450 \mu \mathrm{m}$ and no aggregation between particles was observed.

\section{Particle agglomeration by $\mathrm{LbL}$}

Cross-linked chitosan particles previously produced were placed into a cubic shaped mesh, with $1 \mathrm{~cm}$ side, to prevent them from moving during the dipping process, but mostly to serve as a mold for particles agglomeration, once it will determine the final shape of the scaffold.

The polyelectrolyte solutions were prepared following the same procedure described above. Since chitosan particles exhibit positively charged surface in acidic solution, multilayer assembly must start now with alginate, which is negatively charged. Thus, now multilayers with integer number of DL have chitosan in the outermost layer. The chitosan particles were then first dipped in the alginate solution for $15 \mathrm{~min}$, followed by two periods of $5 \mathrm{~min}$ in purified water. Then, the particles were dipped in the chitosan solution for $15 \mathrm{~min}$, followed by two periods of $5 \mathrm{~min}$ in purified water. After these six steps, one DL had been assembled. The process was repeated until 10 and 20 DLs were assembled. The 
terminal layer was chosen to be chitosan to avoid surface chemistry influence on the behavior of scaffolds, namely, on cell culture results. The double washing period with water warranted a more complete removal of the excess of polyelectrolytes from the interior of the 3D construct.

\section{Scanning electron microscopy}

The surface morphology of the samples was analyzed via scanning electron microscopy (SEM), equipped with a Leica Cambridge S360 scanning electron microscope (Leica). All samples were precoated with a conductive layer of sputtered gold. All the images were taken at $15 \mathrm{keV}$ with different magnifications.

\section{Atomic force microscopy}

An atomic force microscope was used in the characterization of the surface topography of the two-dimensional (2D) multilayers. The microscope used a Nanoscop Scanning Probe Microscope System III (Multimode Digital Instruments). The scanning tips were RTESP from Veeco, and the scanning was performed in the tapping mode, $1 \mathrm{~Hz}$ frequency. $5 \times 5 \mu \mathrm{m}$ scans were performed in different locations.

\section{Microcomputed tomography}

The scaffolds were scanned using a microcomputed tomography (micro-CT) for morphological and morphometric characterization. Micro-CT was carried out with a highresolution micro-CT SkyScan 1072 scanner (Skyscan Kontich) using a resolution of pixel size of $6.69 \mu \mathrm{m}$ and integration time of $1.9 \mathrm{~ms}$. The X-ray source was set at $33 \mathrm{keV}$ of energy and $197 \mathrm{~mA}$ of current. The output format for each sample was 350 serial $1024 \times 1024$ bitmap images. This was used for morphometric analysis, namely, porosity, interconnectivity, and mean pore size (software CT Analyser v1.5.1.5; Skyscan). Interconnectivity was calculated according to the following formula: $I=\left[\left(V_{\text {totalpore }}-V_{\text {disconnectedpore }}\right) / V_{\text {totalpore }}\right] \times 100$, where the volume of the disconnected pore stands for the disconnected pore volume, which was defined to be higher than $50 \mu \mathrm{m} .{ }^{36}$

\section{Contact angle measurements}

The wettability of the studied surfaces was assessed by contact angle measurements. Measurements were performed using a goniometer OCA15+ (DataPhysics) using $3 \mu \mathrm{L}$ drops of $\mathrm{H}_{2} \mathrm{O}$ HPLC. The water contact angle was determined by the sensile drop method adjusting the drop contour to the Laplace equation. The presented data were calculated using the final averaged values of the contact angles, measured using the Sca20 software.

\section{Cell culture}

Two different cell lines were used in this study, the ATDC5 cells, a murine chondrocytic cell line, and L929 cells, a murine fibroblast cell line, both obtained from European Collection of Cell Cultures. The L929 cells were cultured in Dulbecco's modified Eagle's medium (DMEM; Invitrogen) with phenol red supplemented with $10 \%$ of fetal bovine serum (FBS; Invitrogen) and 1\% antibiotic/anti- micotic (Gibco) in a humidified atmosphere with $5 \% \mathrm{CO}_{2}$ and at $37^{\circ} \mathrm{C}$. The ATDC5 cells were cultured in DMEM nutrient mixture F-12 (DMEM F-12, includes L-glutamine; Invitrogen) supplemented with 10\% FBS (Invitrogen) and $1 \%$ antibiotic/antimicotic (Gibco) in a humidified atmosphere with $5 \% \mathrm{CO}_{2}$ and at $37^{\circ} \mathrm{C}$. The medium was changed every 2 days.

\section{Cell seeding}

The samples were placed in contact with cells during different culturing time periods (1,3, and 7 days) to identify differences in attachment and proliferation. Three samples per material per culturing time were studied. Cells were detached from culture flasks using a $0.25 \%$ trypsin/EDTA solution (Sigma). The cells were then concentrated by centrifugation at $1200 \mathrm{rpm}$ for $5 \mathrm{~min}$ and resuspended in culture medium. A total of $1.5 \times 10^{5}$ cells for $2 \mathrm{D}$ samples and $2 \times 10^{5}$ cells for scaffolds were used for each sample. Samples were put under UV light for $60 \mathrm{~min}$, and then they were placed into 24-well culture suspension plates and then washed in a $1 \mathrm{~mL}$ phosphate-buffered saline (PBS) for $30 \mathrm{~min}$. Cells samples previously prepared were then seeded in each sample. Samples were then placed into the incubator for $2 \mathrm{~h}$. After that period, the culture medium was added to each sample and plates placed into the incubator for the established periods of time.

\section{DNA quantification}

After each culturing period, samples were washed in sterile PBS twice and then placed into a $15 \mathrm{~mL}$ Falcon tube, after which $1 \mathrm{~mL}$ of ultrapure water was added. The tubes were placed in a bath at $37^{\circ} \mathrm{C}$ for $60 \mathrm{~min}$. The tubes were then placed in the $-80^{\circ} \mathrm{C}$ freezer for at least $24 \mathrm{~h}$ before use. At the time of use, a $1 \times \mathrm{TE}$ solution was prepared. Quantification standard solutions of $\lambda$ DNA (component C) $0,0.2,0.5,1$, and $2 \mu \mathrm{g} / \mathrm{mL}$ were prepared to obtain a standard curve. A 200fold PicoGreen dsDNA quantification kit (component A) was prepared using $1 \times \mathrm{TE}$ as the solvent. Before starting the assay, samples were sonicated at $37^{\circ} \mathrm{C}$ for $20 \mathrm{~min}$. Samples and control standards were vortexed and $28.8 \mu \mathrm{L}$ of each, plus $71.2 \mu \mathrm{L}$ of the PicoGreen solution and $100 \mu \mathrm{L}$ of the $1 \times \mathrm{TE}$ buffer were transferred into an opaque 96-well plate. Triplicates were made from each sample and control standards. The plate was read using a microplate ELISA reader (BioTek) using an excitation of $485 / 20 \mathrm{~nm}$ and an emission wavelength of $528 / 20 \mathrm{~nm}$.

\section{MTS viability test}

After each culturing period, samples were washed in sterile PBS twice and placed in a new 24-well plate. Samples were then washed for a third time. To start the test, $500 \mu \mathrm{L}$ of culture medium without FBS were added to each well containing a sample and to one well without samples to serve as control. After this, $100 \mu \mathrm{L}$ of MTS [3-(4,5-dimethylthiazol-2-yl)-5(3-carboxymethoxyphenyl)-2-(4-sulfophenyl)-2H-tetrazolium] reagent was added to each well containing samples and to the sample control well. The ratio for the culture medium/ MTS is 5:1. Incubation took place for $3 \mathrm{~h}$ at $37^{\circ} \mathrm{C}$ and $5 \% \mathrm{CO}_{2}$ atmosphere. In the end of the incubation period, $100 \mu \mathrm{L}$ volumes in triplicate was transferred from each well to a 
96-well plate. The plate was read using a microplate ELISA reader (BioTek) at $490 \mathrm{~nm}$ absorbance mode.

\section{Statistical analysis}

The quantification of dsDNA and MTS was performed in three separate sets of experiments with three replicates for each material. All data were averaged and the mean \pm standard deviation was reported. The data were statistically compared by a two-sample assuming equal variances analysis using a Student's $t$-test. In all statistical evaluations, $p<0.05$ was considered as statistically significant.

\section{Results and Discussion}

\section{Multilayer surface characterization}

The effect of substrate surface characteristics on cell adhesion and differentiation has been the subject of a number of investigations. ${ }^{37-42}$ The LbL methodology allows the production of surfaces exhibiting different properties, in- cluding roughness and stiffness. The effect of the substrate characteristics on cell adhesion had been checked for several different cell types, such as fibroblasts, skeletal muscle cells, and myoblasts. ${ }^{43-46}$

In this work, multilayers of chitosan and alginate have been prepared in glass coverslips by using the LbL methodology, obtaining assemblies with 10 and 20 DLs (alginate as terminal layer) and $101 / 2$ and $201 / 2$ DL (chitosan as terminal layer).

Surface topography of multilayers was characterized using atomic force microscopy (AFM). The 3D micrographs are illustrated in Figure 1 and allowed the determination of surface roughness: $23 \pm 7 \mathrm{~nm}$ on the $10 \mathrm{DL}$ film, $29 \pm 8 \mathrm{~nm}$ on the $101 / 2$ DL film, $76 \pm 4 \mathrm{~nm}$ on the 20 DL film and $81 \pm 15 \mathrm{~nm}$ on the $201 / 2$ DL film. It is observed that surface roughness increases with increasing number of layers. Moreover, films having chitosan as terminal layer tended to have a slightly rougher surface than alginate-terminated films, which is attributed not only to the addiction of one polymer layer but mainly to the polymers surface characteristics, once chitosan

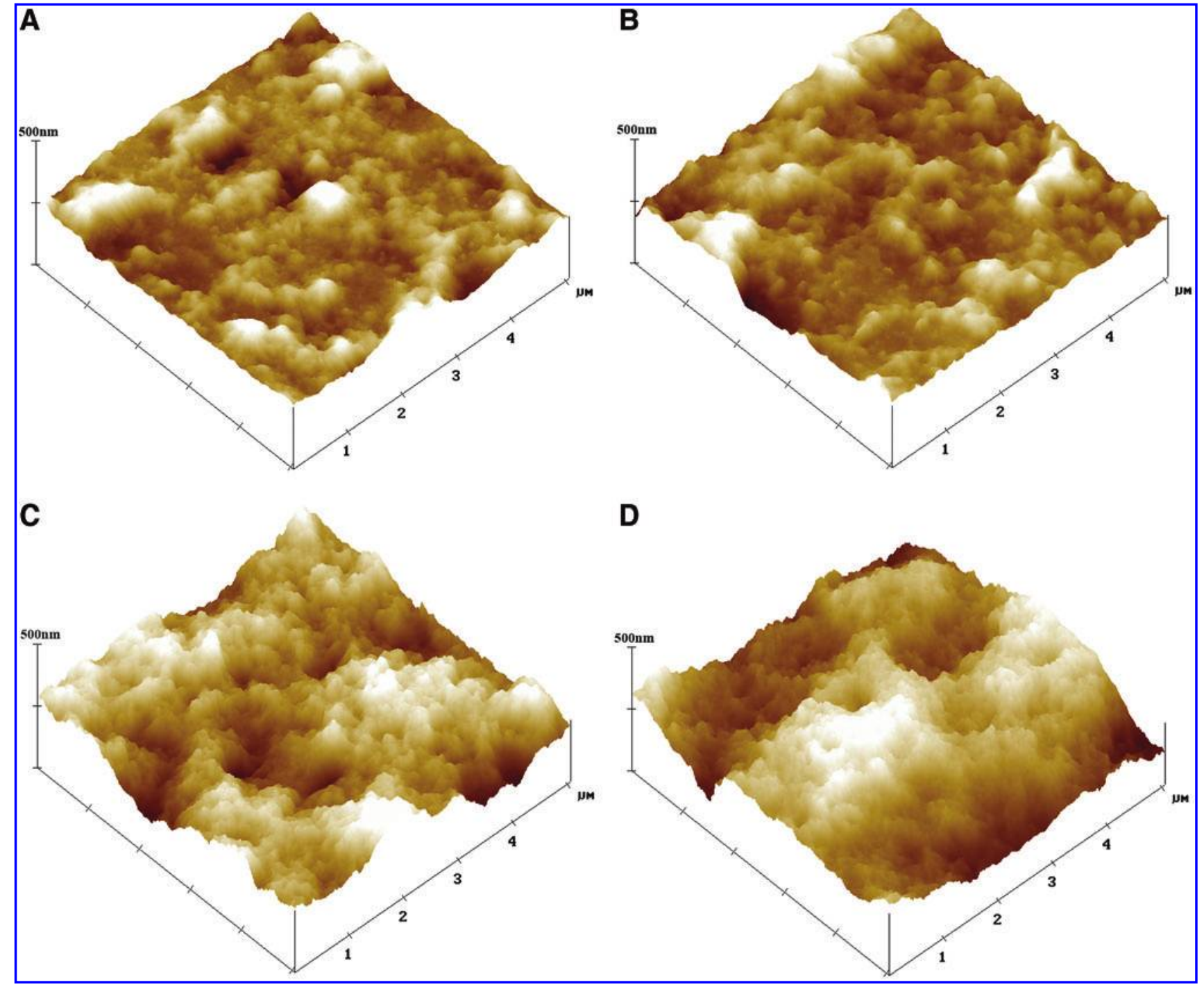

FIG. 1. Atomic force micrographs showing the surface morphology of (A) 10 double layer (DL), (B) 101/2 DL, (C) 20 DL, and (D) $201 \frac{1}{2}$ DL films. Height scale $(0-500 \mathrm{~nm})$ and scan size $(5 \times 5 \mu \mathrm{m})$ are the same for all samples to allow better comparison. Color images available online at www.liebertonline.com/tea 


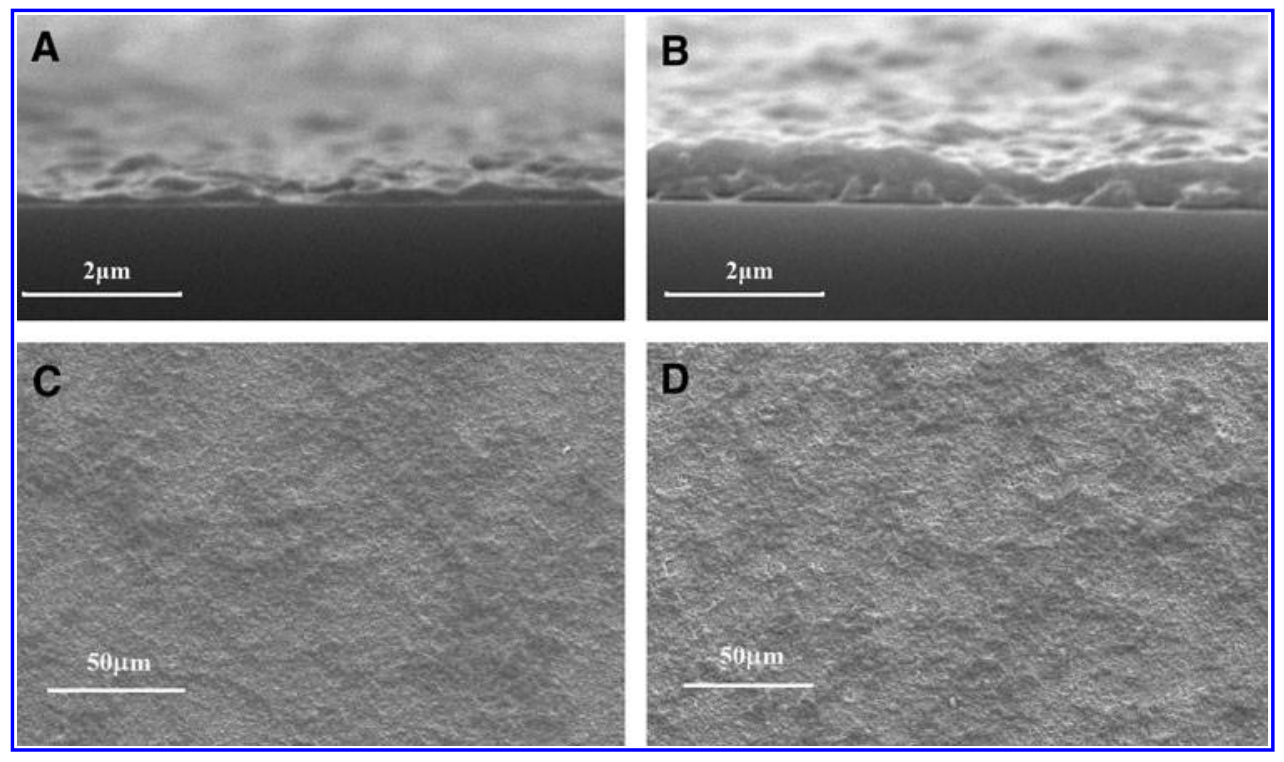

FIG. 2. Scanning electron micrographs showing the film thickness (A, B) and film roughness $(\mathbf{C}, \mathbf{D})$ of $10 \frac{1}{2}$ and $201 / 2$ DL, respectively. The scale bar in images (A) and (B) corresponds to $2 \mu \mathrm{m}$, and in images (C) and (D) corresponds to $50 \mu \mathrm{m}$.

terminated films also present more variability (higher standard deviation).

Surface topography was also characterized by SEM analysis (Fig. 2C, D) and surface roughness was observed to increase with increasing number of layers, confirming the observations made by AFM. Cross sections of assembled multilayers were also observed by SEM analysis, with the results for $101 / 2$ and $201 / 2$ DL films being depicted in Figure $2 \mathrm{~A}$ and B, allowing the assessment of film thickness. It is observed that thickness increases with increasing number of layers, from $200 \pm 70 \mathrm{~nm}$ of $10 \frac{1}{2}$ DL film to $800 \pm 200 \mathrm{~nm}$ of $201 / 2$ DL film. The thickness of 10 and 20 DL films were also observed (data not shown), being $180 \pm 70 \mathrm{~nm}$ and $720 \pm 140 \mathrm{~nm}$, respectively.

These observations show a nonlinear growth for this multilayer, in the described experimental conditions. Linear growth of multilayers using the same polyelectrolytes has also been observed, but when assembled onto a different substrate and under different experimental conditions (namely $\mathrm{pH}$ and dipping time). ${ }^{34}$

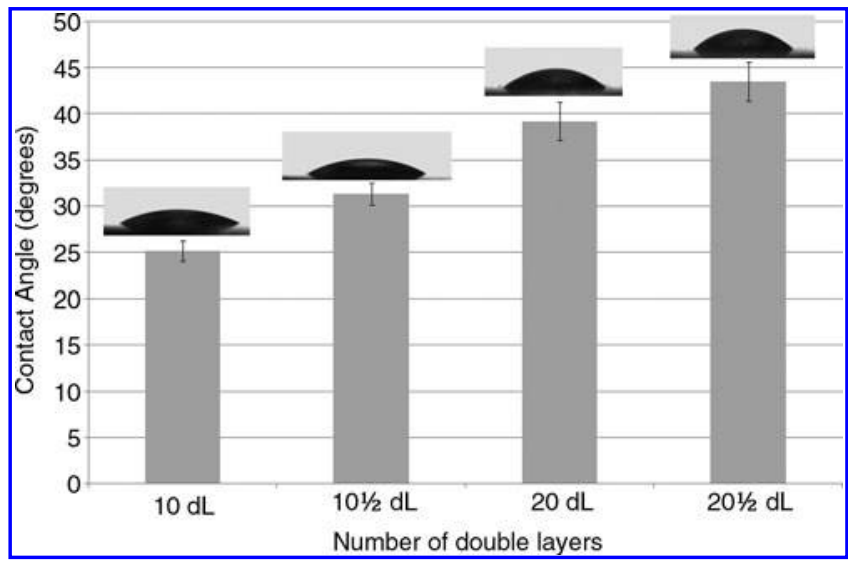

FIG. 3. Contact angle measurements for the different number of DLs. Representative micrographs showing the water drop profiles are shown for each condition.
Surface properties of prepared multilayers were also characterized by contact angle measurements, to assess surface energy and wettability. Such parameters significantly influence the way cells adhere and proliferate on a substrate. ${ }^{47-49}$ The obtained results are illustrated in Figure 3 and shown an increase of contact angle from $25^{\circ}$ to $43^{\circ}$ with increasing number of layers, which can be explained by the increase in surface roughness as can be understood based on the Wenzel model. ${ }^{50}$

\section{Cell behavior in multilayers}

Cell performance onto the assembled multilayers was assessed with two types of cell lines: L929, a murine fibroblast cell line, and ATDC5, a murine chondrocytic cell line. The L929 cells were seeded in all the different sample configurations $\left(10,10 \frac{1}{2}, 20\right.$, and $201 \frac{1}{2}$ DL modified glass coverslips) to test cell behavior in surfaces with different roughness and chemical cues. Samples were analyzed by SEM after 7 days of culture and the respective images are illustrated in Figure 4, where cell attachment and morphology can be observed. Image analysis showed that cell morphology was very similar between samples and thus surface roughness and chemistry appeared to have no visible effect in cell morphology and attachment, as expected from the results reported in literature, where L929 fibroblasts are known to attach on both smooth and rough surfaces $^{51}$ and accordingly to our previous findings on L929 cells culture onto multilayers of chitosan and alginate. $^{52}$

The proliferation of L929 cells was also evaluated and Figure 5A depicts the amount of DNA measured after 1, 3, and 7 days of culture. At day 1 of culture, the DNA quantity increased with increasing number of DLs. At day 3 of culture, the differences between samples DNA amount increased and kept the same tendency showed in day 1 . At day 7 of culture, DNA quantity also increased with increasing number of DLs. The DNA quantity generally increased in all samples for all times of culture, showing the progressive proliferation of cells into the modified glass slides, with more 
FIG. 4. Scanning electron micrographs showing L929 cells in (A) $10 \frac{1}{2}$ DL sample and (B) $201 / 2$ DL sample 7 days after seeding. The scale bar on the down left corner of each image corresponds to $20 \mu \mathrm{m}$.

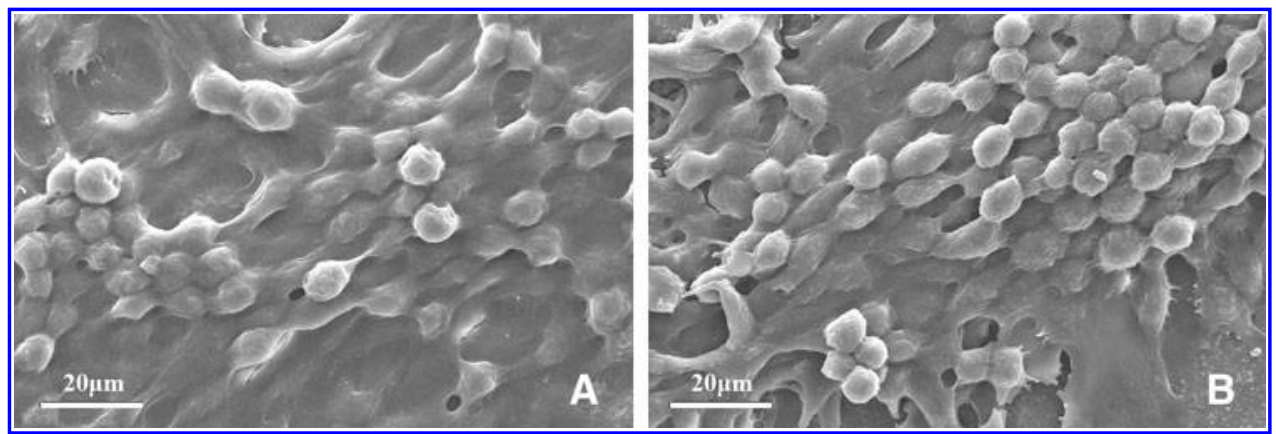

significant proliferation with higher number of polymer layers and higher surface roughness.

The viability of L929 cells was evaluated after the direct contact assay by using the MTS test. The obtained results are illustrated in Figure 5B that depicts the respective absorbance values for $490 \mathrm{~nm}$ for the experiments after 1,3, and 7 days of culture. At day 1 of culture, cell viability increased with increasing number of DLs, independent on the outermost layer. However, at day 3 of culture, a new tendency was observed. Higher cell viability was observed when chitosan was the terminal layer (101/2 and $201 / 2$ DL), when comparing with multilayers where alginate was the terminal layer (10
FIG. 5. DNA quantity (A) and cell viability, determined by using MTS test, measuring the absorbance at $490 \mathrm{~nm}$ (B) in L929 cells direct contact assay with 10, 101/2, 20 , and $201 / 2$ DL in 1,3 , and 7 days of culture. Data represent mean and error bars \pm standard deviation, $n \geq 9$. *Significant difference when compared to 10 DL sample. \#Significant difference when compared to $10 \frac{1}{2}$ DL. ${ }^{* *}$ Significant difference when compared to 20 DL.

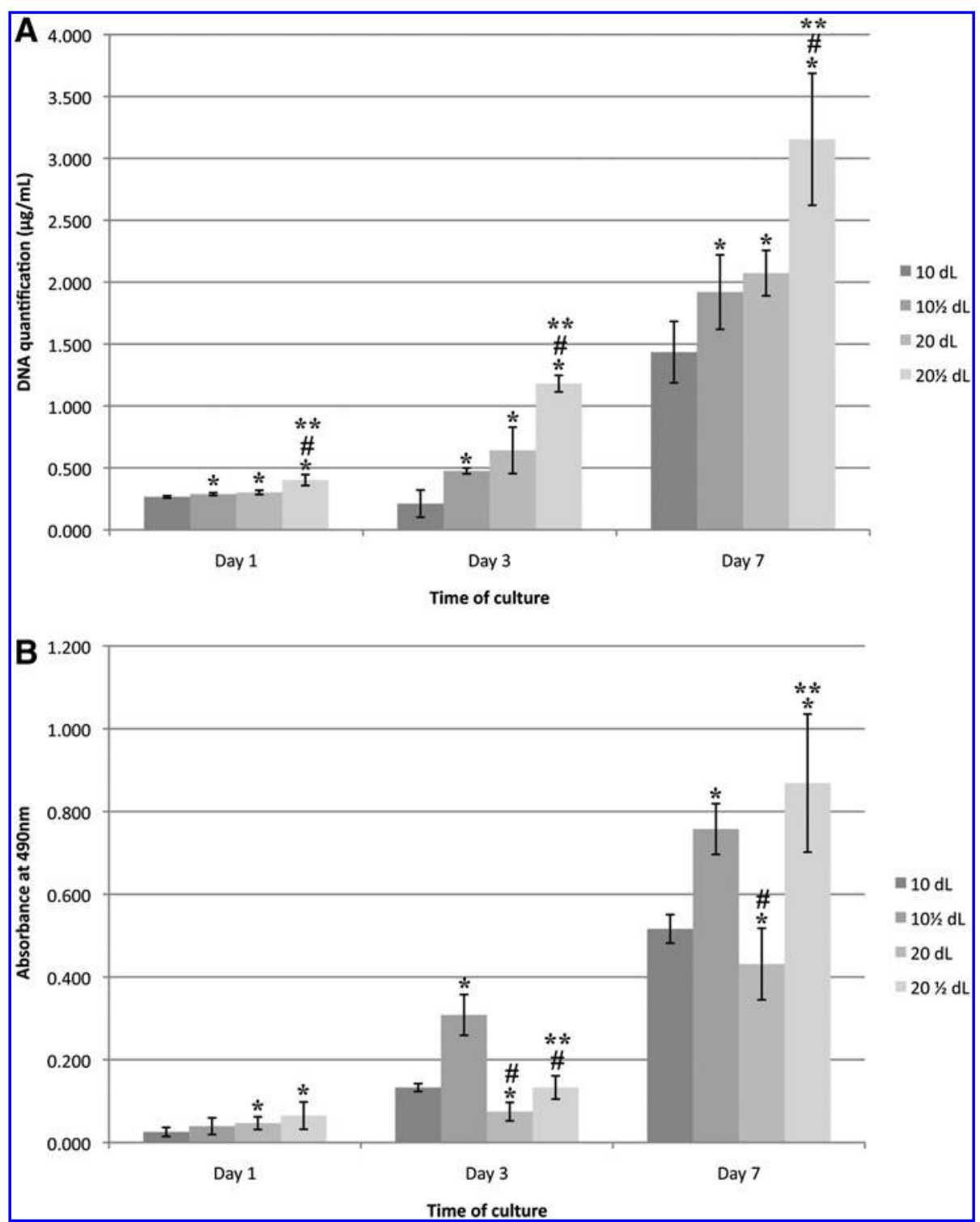


and 20 DL). Thus, a preference of L929 cells for chitosan surfaces seems to exist. At day 7, cell viability increased in all samples and the same tendency observed in day 3 was maintained. Correlating cell proliferation and cell viability data, it was concluded that L929 have a preference for chitosan surface chemistry rather than alginate surfaces, which is in agreement with other studies related to fibroblast adhesion that have suggested that surface amine groups enhanced cell attachment and proliferation..$^{53}$

Cell behavior onto assembled multilayers was also assessed using a murine chondrocytic cell line, ATDC5, since surface roughness is known to play a major role on chondrocytes attachment. ${ }^{54-56}$ SEM images of glass coverslips modified with $10,10^{1 / 2}, 20$, and $20^{1 / 2}$ DL of chitosan and alginate were obtained, being illustrated in Figure 6 with the images of (Fig. 6A) 10 DL and (Fig. 6B) 20 DL films obtained 7 days after ATD5 cells seeding. The images show significant differences in cell morphology and cells attachment. Onto 10 and 101/2 DL films, cells were highly spread on the surface and reached confluence 7 days after seeding. In the other hand, cells in the 20 and 201/2 DL samples were agglomerated in small cell populations and presented a more round morphology.

The proliferation of ATDC5 cells was also evaluated. Figure 7A shows the amount of DNA after 1, 3, and 7 days of culture. The behavior of the ATDC5 cells found for the different surfaces is very distinct of the data obtained with the L929 cells. At day 1 of culture, the number of adhered cells was significantly different in 10 and $10 \frac{1}{2}$ DL samples when compared with $20 \frac{1}{2}$ DL samples. At day 3 , the mentioned differences are more pronounced and a tendency seems to be forming: DNA quantity decreases with increasing number of layers. The 10,101/2, and 20 DL samples registered a significant increase in DNA quantity when compared with day 1 . However, 201/2 DL samples registered a reduction in the DNA quantity when compared with day 1 . The $201 / 2$ DL samples were the ones with the rougher surface and that is thought to be the main reason for the reduced cell attachment. ${ }^{54-56}$ It must be noted that culture medium was changed every 2 days, so cells that could not successfully attach to the materials surface in the first 2 days were removed. After 7 days of culture, the tendency observed after 3 days of culture is confirmed, with DNA quantity decreasing with increasing number of layers. On the other hand, DNA quantities increased in all samples when compared with day 3 . Thus, cells growth in all multilayers studied, but the increasing number of layers seems to hinder that growth, being more significant when chitosan is the terminal layer $\left(10^{1 / 2}\right.$ and $201 / 2$ DL).

Besides proliferation, ATDC 5 cell viability was also evaluated and the results are depicted in Figure 7B, as the absorbance values for $490 \mathrm{~nm}$ after reaction of MTS reagent with samples previously cultured during 1,3 , and 7 days. After 1 day of culture, there were significant differences in viability between some samples, but just after 3 days of culture a tendency arises: it was registered a decrease in cell viability with the increasing number of layers, with significant differences between all samples. After 7 days of culture, it is confirmed the decrease of cell viability with increasing number of layers, despite the continuous increase in cell viability with culture days.

From results obtained for cell proliferation and viability, it can be stated that multilayers with alginate as terminal layer and rendering smother surfaces are more adequate for ATDC 5 cells attachment and proliferation. Equivalent results on the effect of surface roughness have been observed with titanium, by Boyan, Schwartz, and collaborators. ${ }^{54-56}$

\section{Scaffold assembly by particle agglomeration}

It has been hypothesized in the present work that porous scaffolds could be prepared by particle agglomeration by using polyelectrolyte multilayers (PEM) as agglomeration agent. To assess that hypothesis, chitosan particles have been prepared, put together in a mesh mold, and immersed consecutively in polyelectrolyte solutions as described with detail in the experimental section. After the deposition of 10 or 20 DL using the LbL technique, chitosan particles were successfully assembled into porous scaffolds, with particles being coated with multilayers that acted as a joining force. This kind of particle linkage allowed the particle agglomeration without the use of non-natural chemicals and proceeded under mild conditions. The 3D structure was registered using micro-CT, illustrated in Figure $8 \mathrm{~A}$ and $\mathrm{C}$ and porosity and pores interconnectivity were evaluated from analysis of multiple cross-sectional images (illustrated in Fig. 8B), by using software CT Analyser v1.5.1.5, Skyscan. One can conclude that the method proposed enable to produce geometrically stable scaffolds even using 10 DL coatings. Porosity and interconnectivity were calculated for both 10 and 20 DL samples. The porosity was determined to be $42 \%$ and $39 \%$ for 10 and 20 DL samples, respectively. The interconnectivity was $36 \%$ and $42 \%$ to 10 and 20 DL samples, respectively.

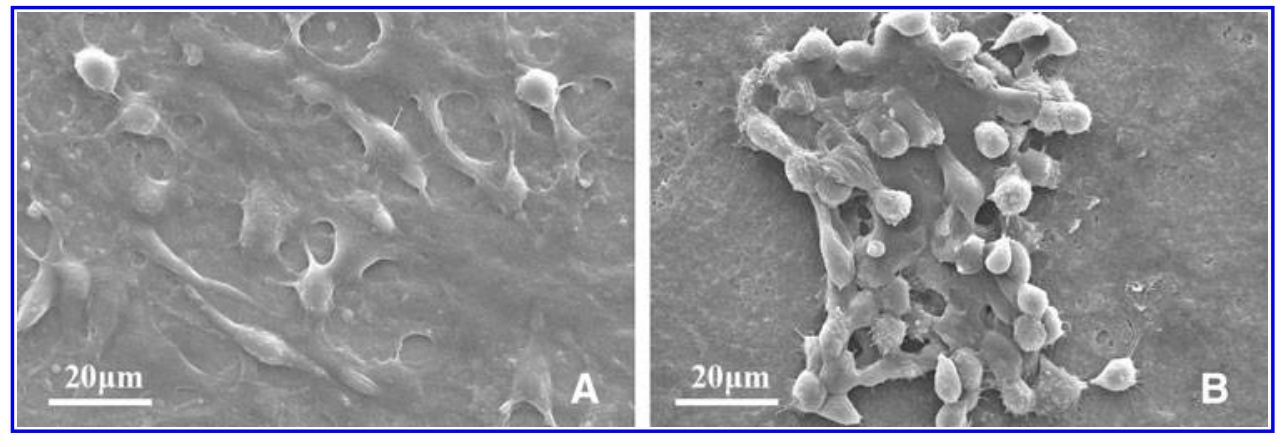

FIG. 6. Scanning electron micrographs showing ATDC5 cells in (A) 10 DL sample and (B) 20 DL sample 7 days after seeding. The scale bar on the down left corner of each image corresponds to $20 \mu \mathrm{m}$. 
FIG. 7. DNA quantity (A) and cell viability, determined by using MTS test, measuring the absorbance at $490 \mathrm{~nm}$ (B) in ATDC5 cells direct contact assay with 10 , $101 / 2,20$, and $201 / 2$ DL in 1,3 , and 7 days of culture. Data represent mean and error bars \pm standard deviation, $n \geq 9$. *Significant difference when compared to 10 DL sample. \#Significant difference when compared to $101 / 2$ DL. **Significant difference when compared to $20 \mathrm{DL}$.

FIG. 8. Microcomputed tomography (micro-CT) from the 10 DL assembled chitosan particle scaffolds. (A) Mesh rendered 3D structure. (B) Cross-sectional representative views of the scaffold showing porosity and interconnection between the particles. (C) Lateral image of the scaffold in the micro-CT support.
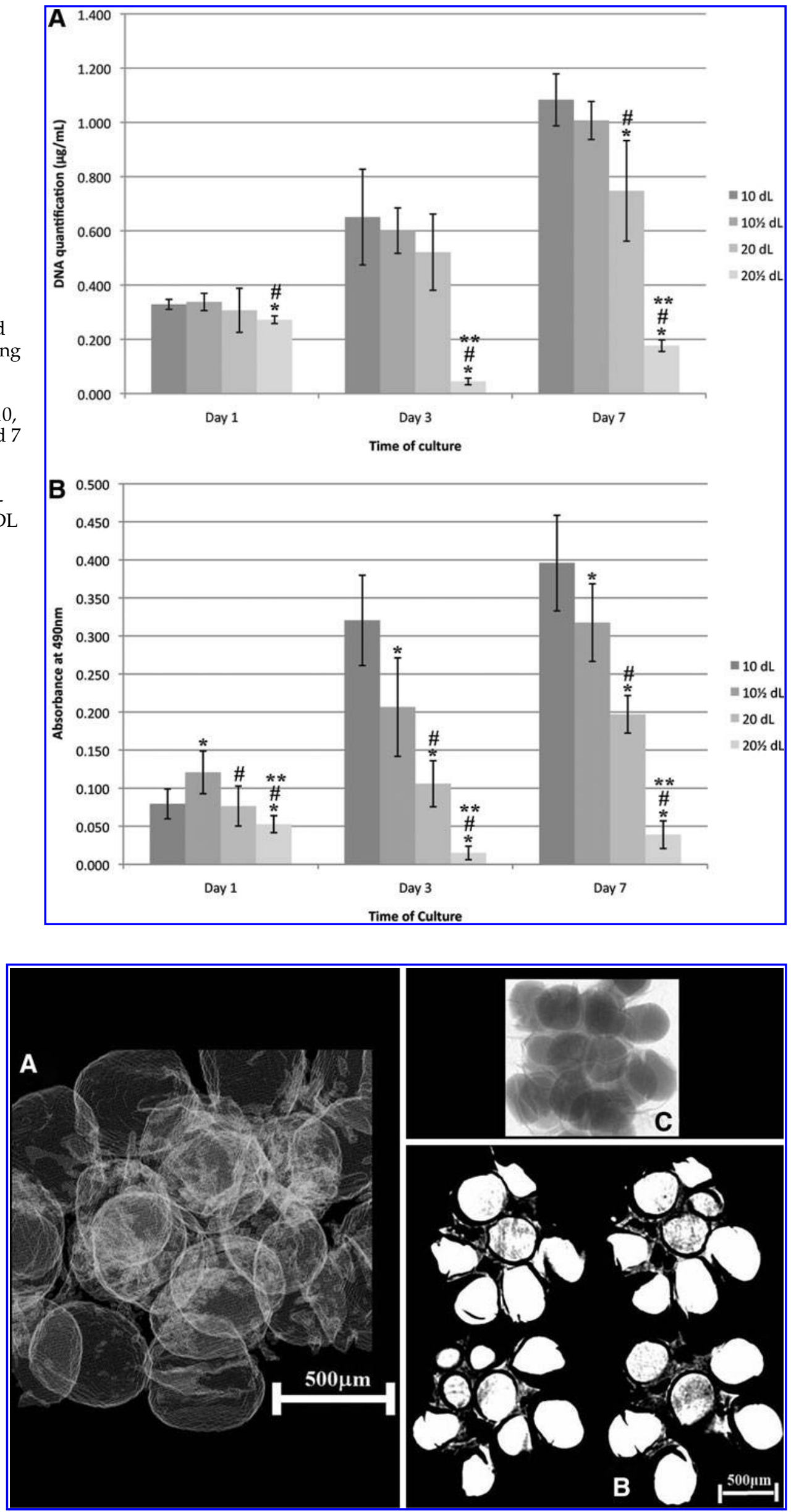
Scaffolds structure showed in Figure 8 corresponds to a dry sample. When in solution, particles became hydrated and grew in size to about $900 \mu \mathrm{m}$ diameter. It is not expected that porosity size would be significantly affected by this particle growth, since all the structure will swell. Regarding mean pore size, it may increase since pores correspond to the empty spaces between spheres and the sphere size is increasing (and so the space between them). However, the swelled structure will have a soft structure and thus some variability can occur in pore sizes.

It should be stressed that the final shape of the scaffold will be determined by the mold used to assemble the particles. As the particles are easily packed into any geometry, one can have a great control on the final architecture of the construct.

\section{ATDC5 cells behavior in assembled scaffolds}

The performance of agglomerated particles as scaffold was assessed by culture of ATDC5 cells. In 2D cell culture studies (previous sections), these cells have shown to be sensitive to both surface chemistry and roughness. Figure 9 shows SEM micrographs of agglomerated chitosan particles after 7 days of culture with ATDC5 cells. Cell attachment in scaffolds surface can be observed in Figure 9B and C.

DNA quantification on the two different types of scaffolds samples (10 and $20 \mathrm{DL}$ ) was performed to assess cell proliferation and the results are illustrated in Figure 10A. After 1 day of culture, DNA quantity in the 10 DL scaffolds was higher than the quantity in the 20 DL scaffolds. After 3 days of culture, DNA quantity for the 10 DL scaffolds increased in a small amount, whereas in the 20 DL scaffolds decrease to about half of the previous time point (day 1). After 7 days of culture, DNA quantity increase significantly in both 10 and 20 DL scaffolds, but being much higher in 10 DL scaffolds, thus showing that cell proliferation decreases with increasing number of DLs.

Cell viability in both 10 and 20 DL scaffolds was also assessed by using the MTS test, and the obtained results are shown in Figure 10B as absorbance at $490 \mathrm{~nm}$ after 1, 3, and 7 days of culture. It is observed that cell viability in $10 \mathrm{DL}$

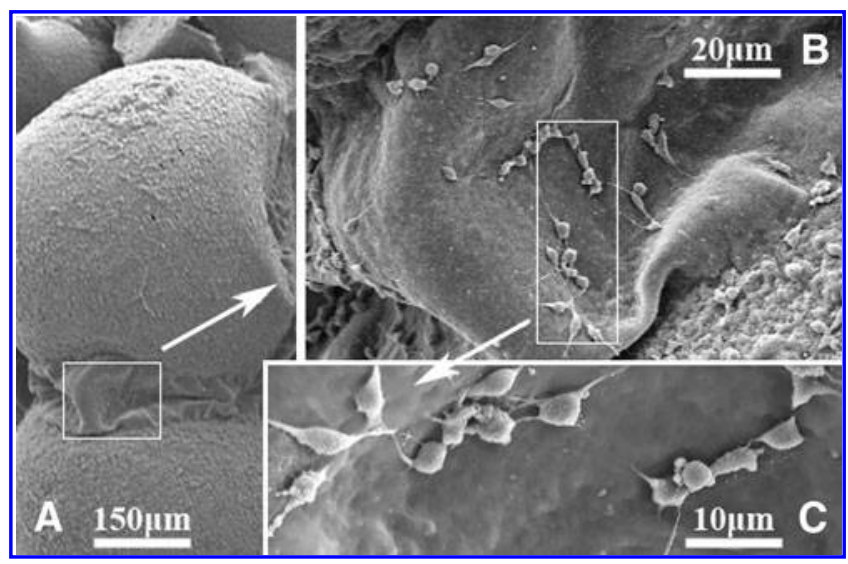

FIG. 9. Scanning electron micrographs of 20 DL scaffold showing (A) chitosan particles agglomerated into a scaffold by multilayers. (B) Multilayer connection between two chitosan particles. (C) Adherent ATDC5 cells in the scaffolds surface.

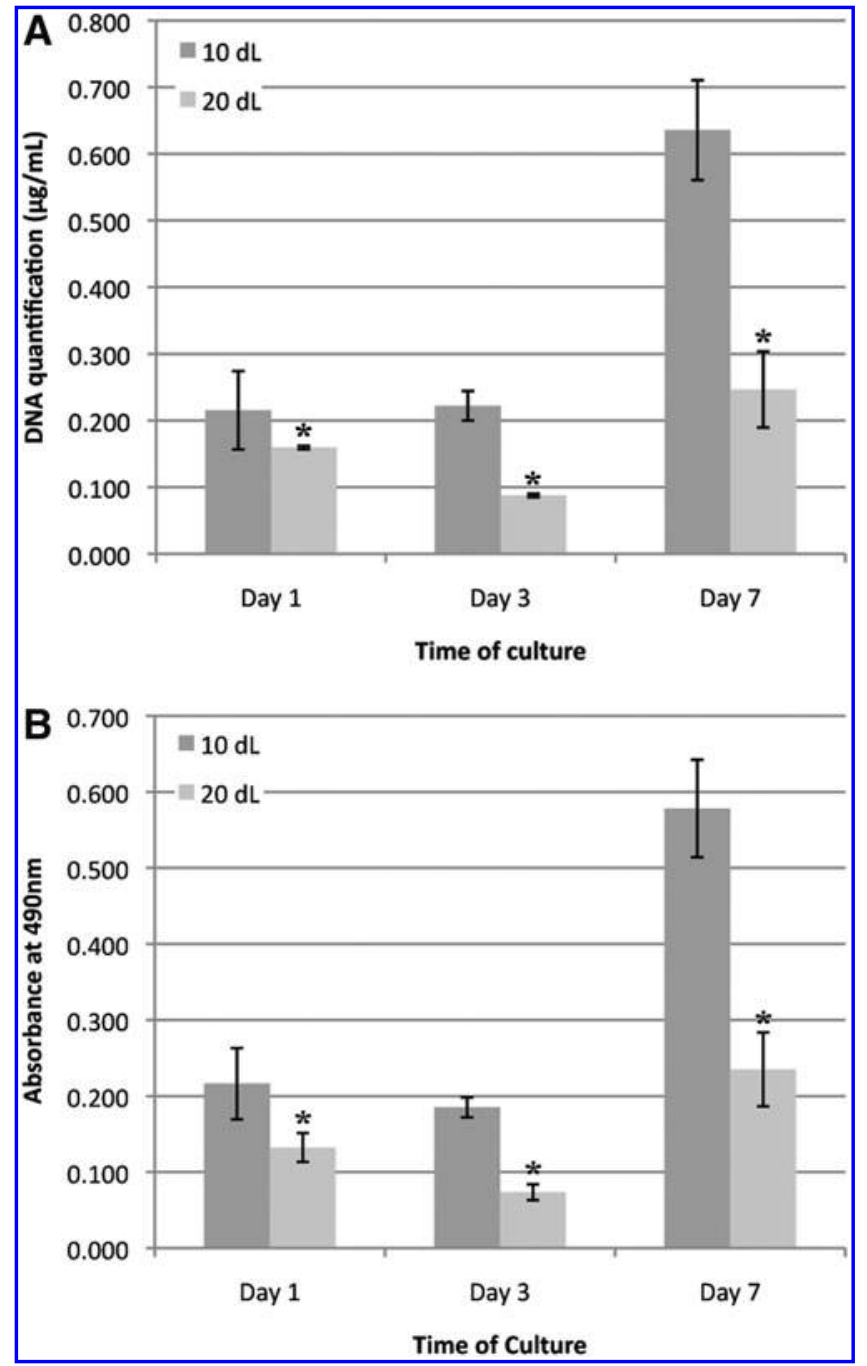

FIG. 10. DNA quantity (A) and cell viability, determined by using MTS test, measuring the absorbance at $490 \mathrm{~nm}$ (B) in ATDC5 cells direct contact assay with 10 and 20 DL scaffolds in 1, 3, and 7 days of culture. Data represent mean and error bars \pm standard deviation, $n \geq 9$. *Significant difference when compared to 10 DL sample.

scaffolds was higher than in 20 DL scaffolds for all times of culture. After 3 days of culture, cell viability is similar (10 DL scaffold) to or smaller (20 DL scaffold) than the observed after 1 day of culture, but after 7 days of culture, cell viability increased very significantly for both type of samples, being much higher in 10 DL scaffold.

The obtained results of cell proliferation and viability have shown that ATDC5 cells attach and proliferate better in smoother surfaces, with lower number of layers, as had been observed in 2D surfaces.

Thus, it is herein proposed that the results observed with multilayers assembled onto planar substrates may be successfully extrapolated to more complex structures such as multilayers assembled onto 3D porous scaffolds.

\section{Conclusions}

The LbL technique was successfully used to deposit multilayers of chitosan and alginate onto glass coverslips. 
Multilayers of $10,10 \frac{1}{2}, 20$, and 201/2 DL were assembled, following a nonlinear film growth behavior, according with thickness measurements from SEM analysis of film cross sections. Surface roughness increases with increasing number of layers, supporting an increase in water contact angle.

Chitosan particles were successfully produced, further cross-linked with genipin, and assembled into 3D scaffolds using the LbL technique, where polyelectrolyte multilayers act as agglomeration agent, avoiding the use of non-natural and harsh chemicals or processing conditions.

The effect of polyelectrolyte multilayers in cell behavior was assessed with a mouse fibroblast cell line (L929) and with a mouse chondrocytic cell line (ATDC5). L929 cells were observed to attach and proliferate better in chitosanterminated multilayers, whereas ATDC5 cells were observed to attach and proliferate better also in smother surfaces, besides the positive effect of chitosan as terminal layer. This behavior of ATDC5 cells was also observed in porous scaffolds resulting from the chitosan particle aggregation with polyelectrolyte multilayers. It was thus shown that the number of layers and the terminal layer of polyelectrolyte multilayers might have an important role in cell attachment, viability, and proliferation, depending on the cell type.

This study suggests that LbL can be used as a single methodology to prepare scaffolds by particle agglomeration that could be suitable for TE applications. Moreover, optimal configurations of polyelectrolyte multilayers can be determined according to cell type to render scaffolds with tuned surface properties to enhance cells behavior.

\section{Acknowledgments}

This work was partially supported by the Portuguese Foundation for Science and Technology (FCT) through project PTDC/QUI/68804/2006, by the European Union Seventh Framework Programme (FP7/2007-2013) under grant agreement no. NMP4-SL-2009-229292 (Find and Bind), and by FEDER through the POCTEP project 0330_IBEROMARE_1_P. T.H. Silva gratefully acknowledges FCT for his postdoc grant (SFRH/BPD/34704/2007). The authors wish to acknowledge A.R. Duarte for assistance with micro-CT measurements.

\section{Disclosure Statement}

No competing financial interests exist.

\section{References}

1. Hubbell, J.A. Biomaterials in tissue engineering. Nat Biotechnol 13, 565, 1995.

2. Langer, R., and Vacanti, J.P. Tissue engineering. Science 260, 920, 1993.

3. Nerem, R.M., and Sambanis, A. Tissue engineering: from biology to biological substitutes. Tissue Eng 1, 3, 1995.

4. Dalton, P.D., Woodfield, T., and Hutmacher, D.W. SnapShot: polymer scaffolds for tissue engineering (volume 30, p. 701, 2009). Biomaterials 30, 2420, 2009.

5. Hutmacher, D.W. Scaffold design and fabrication technologies for engineering tissues-state of the art and future perspectives. J Biomater Sci Polym Ed 12, 107, 2001.
6. Gomes, M.E., Ribeiro, A.S., Malafaya, P.B., Reis, R.L., and Cunha, A.M. A new approach based on injection moulding to produce biodegradable starch-based polymeric scaffolds: morphology, mechanical and degradation behaviour. Biomaterials 22, 883, 2001.

7. Mooney, D.J., Baldwin, D.F., Suh, N.P., Vacanti, L.P., and Langer, R. Novel approach to fabricate porous sponges of poly(D,L-lactic-co-glycolic acid) without the use of organic solvents. Biomaterials 17, 1417, 1996.

8. Gomes, M.E., Holtorf, H.L., Reis, R.L., and Mikos, A.G. Influence of the porosity of starch-based fiber mesh scaffolds on the proliferation and osteogenic differentiation of bone marrow stromal cells cultured in a flow perfusion bioreactor. Tissue Eng 12, 801, 2006.

9. Mikos, A.G., Bao, Y., Cima, L.G., Ingber, D.E., Vacanti, J.P., and Langer, R. Preparation of poly(glycolic acid) bonded fiber structures for cell attachment and transplantation. J Biomed Mater Res 27, 183, 1993.

10. Hutmacher, D.W. Scaffolds in tissue engineering bone and cartilage. Biomaterials 21, 2529, 2000.

11. Salgado, A.J., Sousa, R.A., Oliveira, J.T., Silva, N., Neves, N.M., Reis, R.L., and Sousa, N. Novel tissue engineering 3D scaffolds for spinal cord injury based on starch/ polycaprolactone blends: development and preliminary assessment of their biological performance. Tissue Eng 13, 1736, 2007.

12. Nam, Y.S., and Park, T.G. Porous biodegradable polymeric scaffolds prepared by thermally induced phase separation. J Biomed Mater Res 47, 8, 1999.

13. Schugens, C., Maquet, V., Grandfils, C., Jerome, R., and Teyssie, P. Polylactide macroporous biodegradable implants for cell transplantation .2. Preparation of polylactide foams by liquid-liquid phase separation. I Biomed Mater Res 30, 449, 1996

14. Whang, K., Thomas, C.H., Healy, K.E., and Nuber, G. A novel method to fabricate bioabsorbable scaffolds. Polymer 36, 837, 1995.

15. Correlo, V.M., Boesel, L.F., Pinho, E., Costa-Pinto, A.R., da Silva, M.L.A., Bhattacharya, M., Mano, J.F., Neves, N.M., and Reis, R.L. Melt-based compression-molded scaffolds from chitosan-polyester blends and composites: Morphology and mechanical properties. J Biomed Mater Res A 91A, 489, 2009.

16. Mikos, A.G., Sarakinos, G., Leite, S.M., Vacanti, J.P., and Langer, R. Laminated 3-dimensional biodegradable foams for use in tissue engineering. Biomaterials 14, 323, 1993.

17. Mikos, A.G., Thorsen, A.J., Czerwonka, L.A., Bao, Y., Langer, R., Winslow, D.N., and Vacanti, J.P. Preparation and characterization of poly(l-lactic acid) foams. Polymer 35, 1068, 1994.

18. Martins, A.M., Santos, M.I., Azevedo, H.S., Malafaya, P.B., and Reis, R.L. Natural origin scaffolds with in situ pore forming capability for bone tissue engineering applications. Acta Biomater 4, 1637, 2008.

19. Borden, M., Attawia, M., Khan, Y., and Laurencin, C.T. Tissue engineered microsphere-based matrices for bone repair: design and evaluation. Biomaterials 23, 551, 2002.

20. Malafaya, P.B., Pedro, A.J., Peterbauer, A., Gabriel, C., Redl, H., and Reis, R.L. Chitosan particles agglomerated scaffolds for cartilage and osteochondral tissue engineering approaches with adipose tissue derived stem cells. J Mater Sci Mater Med 16, 1077, 2005. 
21. Martins, A., Chung, S., Pedro, A.J., Sousa, R.A., Marques, A.P., Reis, R.L., and Neves, N.M. Hierarchical starch-based fibrous scaffold for bone tissue engineering applications. I Tissue Eng Regen Med 3, 37, 2009.

22. Duarte, A.R.C., Mano, J.F., and Reis, R.L. Supercritical fluids in biomedical and tissue engineering applications: a review. Int Mater Rev 54, 214, 2009.

23. Duarte, A.R.C., Mano, J.F., and Reis, R.L. Perspectives on: supercritical fluid technology for 3D tissue engineering scaffold applications. J Bioactive Compat Polym 24, 385, 2009.

24. Cruz, D.M.G., Ivirico, J.L.E., Gomes, M.M., Ribelles, J.L.G., Sanchez, M.S., Reis, R.L., and Mano, J.F. Chitosan microparticles as injectable scaffolds for tissue engineering. J Tissue Eng Regen Med 2, 378, 2008.

25. Chesnutt, B.M., Viano, A.M., Yuan, Y.L., Yang, Y.Z., Guda, T., Appleford, M.R., Ong, J.L., Haggard, W.O., and Burngardner, J.D. Design and characterization of a novel chitosan/nanocrystalline calcium phosphate composite scaffold for bone regeneration. J Biomed Mater Res A 88A, 491, 2009.

26. Shi, X.T., Wang, Y.J., Ren, L., Lai, C., Gong, Y.H., and Wang, D.A. A novel hydrophilic poly(lactide-co-glycolide)/lecithin hybrid microspheres sintered scaffold for bone repair. J Biomed Mater Res A 92A, 963, 2010.

27. Gomes, M.E., Malafaya, P.B., and Reis, R.L. Fiber bonding and particle aggregation as promising methodologies for the fabrication of biodegradable scaffolds for hard tissue engineering. In: Reis, R.L., and San Román, J., eds. Biodegradable Systems in Tissue Engineering and Regenerative Medicine. Boca Raton, FL: CRC Press, 2005, pp. 53-65.

28. Mano, J.F., Silva, G.A., Azevedo, H.S., Malafaya, P.B., Sousa, R.A., Silva, S.S., Boesel, L.F., Oliveira, J.M., Santos, T.C., Marques, A.P., Neves, N.M., and Reis, R.L. Natural origin biodegradable systems in tissue engineering and regenerative medicine: present status and some moving trends. $\underline{\mathrm{J}}$ Soc Interface 4, 999, 2007.

29. Decher, G. Fuzzy nanoassemblies: toward layered polymeric multicomposites. Science 277, 1232, 1997.

30. Decher, G., Hong, J.D., and Schmitt, J. Buildup of ultrathin multilayer films by a self-assembly process. 3. Consecutively alternating adsorption of anionic and cationic polyelectrolytes on charged surfaces. Thin Solid Films 210, 831, 1992.

31. Boudou, T., Crouzier, T., Ren, K.F., Blin, G., and Picart, C. Multiple functionalities of polyelectrolyte multilayer films: new biomedical applications. Adv Mater 22, 441, 2010.

32. Hammond, P.T. Form and function in multilayer assembly: new applications at the nanoscale. Adv Mater 16, 1271, 2004.

33. Tang, Z.Y., Wang, Y., Podsiadlo, P., and Kotov, N.A. Biomedical applications of layer-by-layer assembly: from biomimetics to tissue engineering. Adv Mater 18, 3203, 2006.

34. Alves, N.M., Picart, C., and Mano, J.F. Self assembling and crosslinking of polyelectrolyte multilayer films of chitosan and alginate studied by QCM and IR spectroscopy. Macromol Biosci 9, 776, 2009.

35. Silva, S.S., Motta, A., Rodrigues, M.T., Pinheiro, A.F.M., Gomes, M.E., Mano, J.F., Reis, R.L., and Migliaresi, C. Novel genipin-cross-linked chitosan/silk fibroin sponges for cartilage engineering strategies. Biomacromolecules 9, 2764, 2008.

36. Duarte, A.R.C., Mano, J.F., and Reis, R.L. Preparation of starch-based scaffolds for tissue engineering by supercritical immersion precipitation. J Supercrit Fluids 49, 279, 2009.

37. Hallab, N.J., Bundy, K.J., O'Connor, K., Moses, R.L., and Jacobs, J.J. Evaluation of metallic and polymeric biomaterial surface energy and surface roughness characteristics for directed cell adhesion. Tissue Eng 7, 55, 2001.
38. Lampin, M., WarocquierClerout, R., Legris, C., Degrange, M., and SigotLuizard, M.F. Correlation between substratum roughness and wettability, cell adhesion, and cell migration. J Biomed Mater Res 36, 99, 1997.

39. Lan, M.A., Gersbach, C.A., Michael, K.E., Keselowsky, B.G., and Garcia, A.J. Myoblast proliferation and differentiation on fibronectin-coated self assembled monolayers presenting different surface chemistries. Biomaterials 26, 4523, 2005.

40. Lim, J.Y., Liu, X.M., Vogler, E.A., and Donahue, H.J. Systematic variation in osteoblast adhesion and phenotype with substratum surface characteristics. I Biomed Mater Res A 68A, 504, 2004.

41. Liu, X.M., Lim, J.Y., Donahue, H.J., Dhurjati, R., Mastro, A.M., and Vogler, E.A. Influence of substratum surface chemistry/energy and topography on the human fetal osteoblastic cell line hFOB 1.19: phenotypic and genotypic responses observed in vitro. Biomaterials 28, 4535, 2007.

42. Phillips, J.E., Petrie, T.A., Creighton, F.P., and Garcia, A.J. Human mesenchymal stem cell differentiation on selfassembled monolayers presenting different surface chemistries. Acta Biomater 6, 12, 2010.

43. Mhamdi, L., Picart, C., Lagneau, C., Othmane, A., Grosgogeat, B., Jaffrezic-Renault, N., and Ponsonnet, L. Study of the polyelectrolyte multilayer thin films' properties and correlation with the behavior of the human gingival fibroblasts. Mater Sci Eng C Biomimetic Supramol Syst 26, 273, 2006.

44. Ren, K.F., Crouzier, T., Roy, C., and Picart, C. Polyelectrolyte multilayer films of controlled stiffness modulate myoblast cell differentiation. Adv Funct Mater 18, 1378, 2008.

45. Schneider, A., Francius, G., Obeid, R., Schwinte, P., Hemmerle, J., Frisch, B., Schaaf, P., Voegel, J.C., Senger, B., and Picart, C. Polyelectrolyte multilayers with a tunable Young's modulus: influence of film stiffness on cell adhesion. Langmuir 22, 1193, 2006.

46. Vazquez, C.P., Boudou, T., Dulong, V., Nicolas, C., Picart, C., and Glinel, K. Variation of polyelectrolyte film stiffness by photo-cross-linking: a new way to control cell adhesion. Langmuir 25, 3556, 2009.

47. Faucheux, N., Schweiss, R., Lutzow, K., Werner, C., and Groth, T. Self-assembled monolayers with different terminating groups as model substrates for cell adhesion studies. Biomaterials 25, 2721, 2004.

48. Kennedy, S.B., Washburn, N.R., Simon, C.G., and Amis, E.J. Combinatorial screen of the effect of surface energy on fibronectin-mediated osteoblast adhesion, spreading and proliferation. Biomaterials 27, 3817, 2006.

49. Arima, Y., and Iwata, H. Effect of wettability and surface functional groups on protein adsorption and cell adhesion using well-defined mixed self-assembled monolayers. Biomaterials 28, 3074, 2007.

50. Wenzel, R.N. Resistance of solid surfaces to wetting by water. Ind Eng Chem 28, 988, 1936.

51. Karakecili, A.G., Demirtas, T.T., Satriano, C., Gumusderelioglu, M., and Marletta, G. Evaluation of L929 fibroblast attachment and proliferation on Arg-Gly-Asp-Ser (RGDS)immobilized chitosan in serum-containing/serum-free cultures. J Biosci Bioeng 104, 69, 2007.

52. Martins, G.V., Merino, E.G., Mano, J.F., and Alves, N.M. Crosslink effect and albumin adsorption onto chitosan-alginate multilayered systems: an in-situ study by quartz crystal microbalance with dissipation monitoring. Macromol Biosc: 10, 1444, 2010. 
53. Webb, K., Hlady, V., and Tresco, P.A. Relative importance of surface wettability and charged functional groups on NIH 3T3 fibroblast attachment, spreading, and cytoskeletal organization. J Biomed Mater Res 41, 422, 1998.

54. Boyan, B.D., Hummert, T.W., Dean, D.D., and Schwartz, Z. Role of material surfaces in regulating bone and cartilage cell response. Biomaterials 17, 137, 1996.

55. Boyan, B.D., Lincks, J., Lohmann, C.H., Sylvia, V.L., Cochran, D.L., Blanchard, C.R., Dean, D.D., and Schwartz, Z. Effect of surface roughness and composition on costochondral chondrocytes is dependent on cell maturation state. J Orthop Res 17, 446, 1999.

56. Schwartz, Z., Martin, J.Y., Dean, D.D., Simpson, J., Cochran, D.L., and Boyan, B.D. Effect of titanium surface roughness on chondrocyte proliferation, matrix production, and differentiation depends on the state of cell maturation. J Biomed Mater Res 30, 145, 1996.
Address correspondence to:

Tiago H. Silva, Ph.D.

3B's Research Group-Biomaterials, Biodegradables and Biomimetics

University of Minho

Headquarters of the European Institute of Excellence on Tissue Engineering and Regenerative Medicine AvePark, Zona Industrial da Gandra

S. Cláudio do Barco 4806-909 Caldas das Taipas Portugal

E-mail: tiago.silva@dep.uminho.pt

Received: November 3, 2010

Accepted: June 15, 2011

Online Publication Date: July 22, 2011 